\title{
Efectos de la deficiencia de riboflavina sobre el desarrollo del tejido dentoalveolar, en ratas ${ }^{*}$
}

\author{
Effect of riboflavin defficiency on dentoalveolar tissue development in rats
}

\author{
Juana Delgadillo ${ }^{1}$, Guido Ayala² \\ ${ }^{1}$ Departamento Académico de Ciencias Básicas. Facultad de Odontología, Universidad Nacional Mayor de San Marcos. Lima, Perú. \\ 2 Departamento Académico de Ciencias Dinámicas. Facultad de Medicina, Universidad Nacional Mayor de San Marcos. Lima, Perú. \\ * Trabajo de tesis para optar el título de Doctora en Ciencias de la Salud. Facultad de Medicina, Universidad Nacional Mayor de San Marcos.
}

Resumen

Objetivo: Determinar los cambios histomorfológicos que ocurren en el tejido dentoalveolar de la mandíbula de una progenie de ratas albinas sometidas a dietas con diferentes concentraciones de riboflavina $\left(B_{2}\right)$ e iguales concentraciones de los demás nutrientes, durante el periodo de gestación y lactancia, hasta los 28 días de edad. Diseño: Estudio experimental y analítico. Lugar: Centro de Investigación de Bioquímica y Nutrición Alberto Guzmán Barrón, Facultad de Medicina, Universidad Nacional Mayor de San Marcos, Lima, Perú. Material biológico: Crías de ratas albinas Holtzman. Intervenciones: Se utilizó 60 crías de ratas, que fueron progenie de 16 ratas albinas Holtzman, conformándose 4 grupos con capacidad de reproducción y con diferentes requerimientos de riboflavina: Grupo A ( $100 \%$ de requerimiento de $\left.B_{2}\right)$, Grupo $B\left(50 \%\right.$ de $\left.B_{2}\right)$ Grupo $C\left(25 \%\right.$ de $\left.B_{2}\right)$ y Grupo $D(0 \%$ de $B_{2}$ ). Las crías de cada grupo fueron sacrificadas a los $0,7,14,21$ y 28 días de nacimiento, y se sometió a estudios histológicos los tejidos dentoalveolares de 60 crías. Principales medidas de resultados: Alteraciones en la talla, peso corporal y el tejido dentoalveolar de las ratas crías. Resultados: Microscópicamente, el estudio reveló que la carencia total de riboflavina en la dieta materna $(0 \%$ de $\mathrm{B}_{2}$ ) ocasiona en el tejido dentoalveolar de las crías cambios histomorfológicos, como pérdida de la polaridad nuclear, presencia de microvacuolas y áreas de pseudo-estratificación en ameloblastos y odontoblastos, disminución del número de células del tejido dentoalveolar, aparición de edema en predentina, disminución de las células activas en el caso de fibroblastos y cementoblastos, notable disminución y adelgazamiento de las fibras colágenas y reducción del grosor de las trabeculas óseas. Alteraciones similares pero en menor cuantía se halló en ratas con deficiencia de $B_{2}$. Conclusiones: La carencia total de riboflavina ( $\left.0 \%\right)$ en la dieta materna de las ratas durante la gestación y lactación provoca en el tejido dentoalveolar de las crías una cantidad y calidad menor de ameloblastos, odontoblastos, fibroblastos y cementoblastos; escasa cantidad de fibras colágenas y adelgazamiento del hueso alveolar.

Palabras clave: Manejo nutricional; deficiencia de riboflavina; tejido dentoalveolar.

\begin{abstract}
Objective: To determine histomorphological changes in jaw dentoalveolar tissue in a progeny of albino rats fed with diets containing different concentrations of riboflavin $\left(B_{2}\right)$ and equivalent concentrations of other nutrients, during gestation and lactation and until 28 days of birth. Design: Experimental, longitudinal and analytical. Setting: Alberto Guzman Barron Biochemistry and Nutrition Research Center, Faculty of Medicine, Universidad Nacional Mayor de San Marcos, Lima, Peru. Biologic material: Holtzman albino rats. Interventions: We used 20 Holtzman albino rats (16 females and 4 males) with reproduction capacity and conformed four groups, according to the demands of riboflavin: Group $A\left(100 \%\right.$ of $\left.B_{2}\right)$, Group B ( $50 \%$ of $\left.B_{2}\right)$, Group C ( $25 \%$ of $\left.B_{2}\right)$, and Group D ( $0 \%$ of $\left.B_{2}\right)$. Submitted to ether anesthesia the offspring of each rats group was sacrificed at $0,7,14$, 21 , and 28 days from birth and the dentoalveolar tissues of 60 offspring were subjected to histological examinations. Main outcome measures: Height, weight and dentoalveolar tissue alterations in rat progeny. Results: Deficiency (50\% and $25 \%$ ) and riboflavin total lack $(0 \%)$ caused alterations on offspring growth and development. Microscopy revealed total lack of riboflavin $(0 \%)$ in the mother's diet caused some histomorphological changes in the offspring dentoalveolar tissue, such as loss of nuclear polarity, presence of microvacuoles and areas of pseudo-stratification in ameloblasts and odontoblasts, decrease in the number of cells of the dentoalveolar tissue, edema in predentin, decrease in the number of fibroblasts and cementoblasts active cells, remarkable decrease and slimming of collagen fibers, and reduction of osseous trabeculae's thickness. Conclusions: Total lack of riboflavin in the rat mothers' diet during gestation and lactation causes less quantity and minor quality of ameloblasts, odontoblasts, fibroblasts and cementoblasts, scanty collagenous fibers and slimming of the alveolar bone in the dentoalveolar tissue of their offspring.
\end{abstract}

Key words: Diet therapy; riboflavin deficiency; dentoalveolar tissue.

\section{INTRODUCCIÓN}

La riboflavina (vitamina $B_{2}$ ) es un nutriente esencial que mantiene las funciones del metabolismo energético en condiciones normales, actuando como cofactor en las reacciones enzimáticas, principalmente en el sistema de transporte de electrones. La vitamina $B_{2}$ está presente en los tejidos de los mamíferos bajo las formas de las coenzimas flavin mononucleótido (FMN) y flavin adenin dinucleotido (FAD), actuando como parte de las flavoproteínas que intervienen en el metabolismo de los carbohidratos, grasas y proteínas ${ }^{(1)}$.
Estudios realizados sobre deficiencia de riboflavina en humanos demuestran que esta ocasiona rigidez en la comisura labial y alteraciones en la piel, queilosis, estomatitis angular así como lesiones oculares ${ }^{(1)}$. Sin embargo, las manifestaciones de la deficiencia humana no tienen la especificidad que puede caracterizar a los déficits de algunas otras vitaminas. De forma casi invariable, la deficiencia aparece en combinación con déficit de otras vitaminas hidrosolubles, como la tiamina $\left(\mathrm{B}_{1}\right)$ y la niacina $\left(\mathrm{B}_{3}\right)^{(1,2)}$. Actualmente, se ha reconocido que la deficiencia de riboflavina puede deberse no solamente a una escasa ingesta alimentaria, sino también a enfermedades, fármacos y anomalías endocrinas que pueden interferir en la utilización de la vitamina. Las insuficiencias tiroidea y suprarrenal, los fármacos psicotropos -como la clorpromazina, la imipramina y la amitriptilina-, los agentes quimioterapéuticos anticancerígenos -como la adriamicina- y los antimaláricos -como la quinacrina- inhiben la conversión de riboflavina en sus derivados coenzimáticos activos ${ }^{(3)}$.

El comité conjunto de expertos de la FAO/OMS ha fijado el nivel recomen- 
dado de riboflavina en 0,55 mg por 1000 calorías, para cualquier grupo de edad.

La deficiencia de riboflavina en los animales de experimentación produce alteraciones en el crecimiento, alopecia, alteraciones de la visión, de la mucosa, malformaciones y en muchos casos sangrados. que puede ocasionar la muerte. ${ }^{(4-7)}$. En el Perú, en regiones rurales de la costa y sierra, de condiciones económicas extremas, donde la prevalencia de la desnutrición es alta, se ha observado deficiencia de riboflavina, especialmente en gestantes, lactantes, en niños y adolescentes, contribuyendo a la alteración del crecimiento y desarrollo del humano ${ }^{(8)}$.

La literatura científica no informa sobre los efectos que tendría la carencia de esta vitamina en la gestación, de manera particular en el desarrollo de tejido dentoalveolar del ser en formación, y en el periodo posnatal. Es así que el presente trabajo experimental pretende determinar los efectos sobre el crecimiento y desarrollo del tejido dentoalveolar en animales de experimentación sometidos a dietas con deficiencia o ausencia de riboflavina, desde la gestación hasta el final del destete.

\section{MÉTODOS}

Se realizó un estudio experimental en ratas albinas de raza Holtzman. La muestra estuvo constituida por 60 ratas crías, las que recibieron la dieta en las etapas pre y post natal, hasta los 28 días de nacidas. El trabajo se realizó en el bioterio del Centro de Investigación de Bioquímica y Nutrición de la Universidad Nacional Mayor de San Marcos y en el laboratorio de histología del Instituto de Medicina Tropical de la Facultad de Medicina, de la misma universidad.

En una primera etapa, se seleccionó 20 ratas Holtzman, 16 hembras y 4 machos, todos de 4 meses de edad y con las mismas características, las cuales fueron colocadas en jaulas individuales, durante 5 días, para su apareamiento (4 hembras por cada macho). El diagnóstico de preñez fue mediante la presencia de espermatozoides en el tapón vaginal. Las 16 ratas preñadas, previamente codificadas, fueron ubicadas al azar en grupos de 4, en jaulas individuales, para ser sometidas a la dieta experimental durante la gestación y lactancia. Dichos cuatro grupos dietarios fueron:

- Grupo A (grupo control), conformado por 4 ratas hembras colocadas en jaulas individuales con sus respectivas crías, alimentadas con una dieta normocalórica, normoproteínica y que contenía una concentración de 0,6 mg de vitamina $\mathrm{B}_{2}\left(100 \%\right.$ de $\left.\mathrm{B}_{2}\right)$.

- Grupo B, conformado por 4 ratas hembras colocadas en jaulas individuales con sus respectivas crías, alimentadas con una dieta normocalórica, normoproteínica y que contenía una concentración de 0,3 mg de vitamina $\mathrm{B}_{2}\left(50 \%\right.$ de $\left.\mathrm{B}_{2}\right)$.

- Grupo C, conformado por 4 ratas hembras colocadas en jaulas individuales con sus respectivas crías, alimentadas con una dieta normocalórica, normoproteínica y que contenía una concentración de 0,15 mg de vitamina $B_{2}\left(25 \%\right.$ de $\left.B_{2}\right)$.

- Grupo D, conformado por 4 ratas hembras colocadas en jaulas individuales con sus respectivas crías, alimentadas con una dieta normocalórica, normoproteínica y que careció de vitamina $\mathrm{B}_{2}(0 \mathrm{mg})\left(0 \%\right.$ de $\left.\mathrm{B}_{2}\right)$.

Además, en cada jaula se colocó una ración diaria de 100 gramos de dieta que contenía todos los demás nutrientes, en concentraciones de acuerdo a sus requerimientos ${ }^{(9,10)}$ : caseína $(10 \mathrm{~g})$, metionina $(0,3 \mathrm{~g})$, mezcla de minerales $(4 \mathrm{~g})$, celulosa $(2 \mathrm{~g})$, almidón $(72,2 \mathrm{~g})$, aceite $(10,9 \mathrm{~mL})$, mezcla de vitaminas $(0,1 \mathrm{~mL})$, vitamina E (20 mg). Se controló el consumo de alimento, el peso de las ratas madres, así como, de las crías después del nacimiento, cada 2 días, durante toda la fase experimental.

Se extrajo al azar 3 ratas para ser sacrificadas (previa anestesia), a los 0 , 7, 14, 21 y 28 días, de acuerdo al tiempo de nacimiento, haciendo un total de 60 crías. Después del sacrificio, se obtuvo las muestras de la mandíbula para su estudio. Las muestras de la región mandibular fueron disecadas para ser descalcificadas. Luego, se utilizó la coloración de hematoxilina eosina para observar la población celular (ameloblastos, odontoblastos, fibroblastos, cementoblastos) y con tricrómico de masón para ver fibras colágenas. La observación fue posible mediante el uso del microscopio de luz a diferentes aumentos $(20 \mathrm{x}, 40 \mathrm{x}, 100 \mathrm{x}) \mathrm{y}$ el ocular micrométrico dentro de un área de 1600 micras para la cuantificación celular y la cantidad de fibras colágenas.

Se utilizó la prueba Fisher para probar homogeneidad de varianzas. Para el análisis estadístico, se utilizó la prueba Anova (11). Se presenta los datos en promedios y desviación estándar; se consideró significancia estadística si $p<0,05$.

\section{RESULTADOS}

La información se obtuvo del examen de 140 cortes histológicos, de los cuales 60 correspondieron a tejido óseo mandibular en corte coronal, 60 en corte longitudinal y 20 a fibras colágenas. Se observó diferencias significativas en cuanto a la talla y peso corporal, así como longitud y grosor de mandíbula, entre los diferentes grupos (grupo $\mathrm{A}: 100 \%$ de $\mathrm{B}_{2}$, grupos $\mathrm{B}$ : $50 \%$ de $B_{2}$, grupo $C: 25 \%$ de $B_{2}$, grupo C: $0 \%$ de $\mathrm{B}_{2}$ ).

En cuanto al peso corporal, se halló una diferencia significativa entre el grupo A (promedio de peso $\bar{X}=46,7 \mathrm{~g}$ ) respecto al grupo $\mathrm{D}(\overline{\mathrm{X}}=20,3 \mathrm{~g})$. En la longitud corporal, se observó diferencias significativas en la talla promedio de los grupos de ratas A, B y C $(21,13 \mathrm{~cm} ; 18,80 \mathrm{~cm}$ y $18,77 \mathrm{~cm}$, respectivamente), respecto al grupo D $(14,87 \mathrm{~cm})$.

En cuanto a la longitud mandibular, existieron diferencias significativas entre los grupos $\mathrm{A}(\overline{\mathrm{X}}=15 \mathrm{~mm})$ y $\mathrm{B}(\overline{\mathrm{X}}=13,67$ $\mathrm{mm})$ respecto a los grupos $C(\bar{X}=12,33$ $\mathrm{mm})$ y $\mathrm{D}(\overline{\mathrm{X}}=10,33 \mathrm{~mm})$, siendo mucho más significativa la diferencia entre el grupo control A y el grupo D. En cuanto al grosor mandibular, hubo diferencia significativa entre el grosor de la mandíbula del grupo $\mathrm{A}(\overline{\mathrm{X}}=1,93 \mathrm{~mm})$ respecto al grupo $\mathrm{D}(\overline{\mathrm{X}}=1,43 \mathrm{~mm})$.

Con relación a las características microscópicas generales de los diferentes grupos, los cortes longitudinales y coronales de la mandíbula demostraron que la dieta de $100 \%$ de $\mathrm{B}_{2}$, el grupo A tuvo 


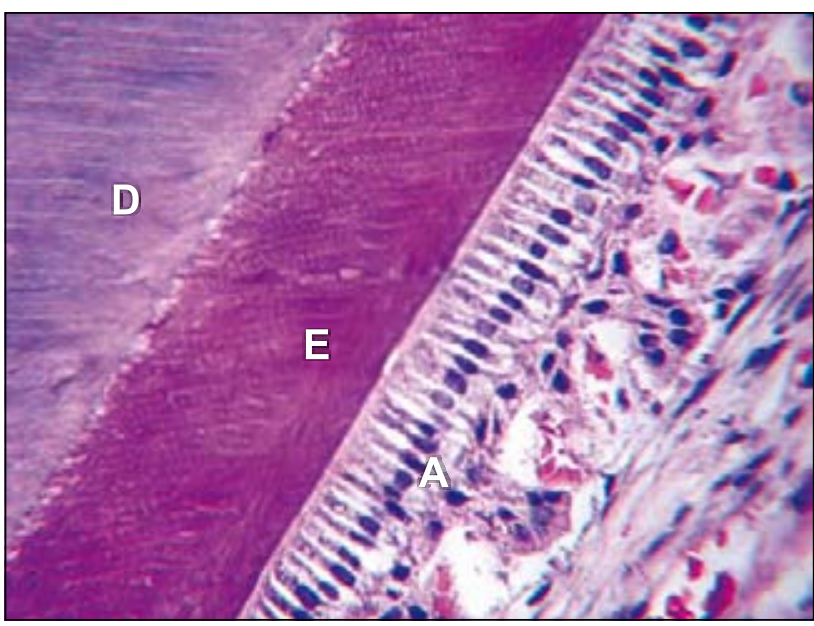

Figura 1. Tejido dentoalveolar con $100 \%$ de riboflavina, a los 7 días. En el corte longitudinal de diente (40X), se ve ameloblastos (A) cilíndricos y columnares con núcleo basal. El esmalte $(E)$ se visualiza como una banda eosinófila acelular que contrasta con la dentina (D) basófila.

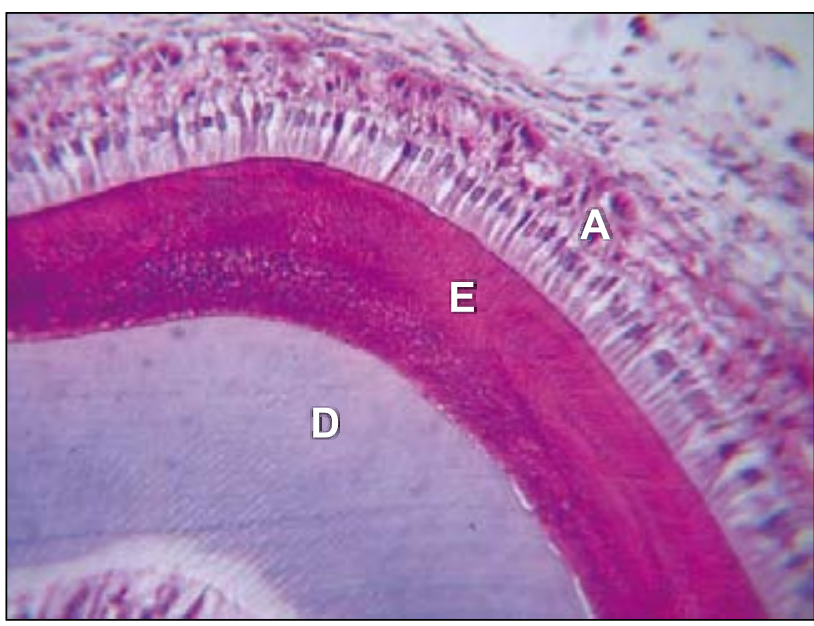

Figura 2. Tejido dentoalveolar con $100 \%$ de riboflavina, a los 21 días. En corte coronal (40X) a nivel de diente, ameloblastos (A) cilíndricos y columnares con núcleo basal; escasas vacuolas. El esmalte $(E)$ se visualiza como una banda eosinófilo acelular que contrasta con la dentina (D) basófila. un trabeculado óseo de grosor variable, el tejido dentoalveolar con una mayor población celular de ameloblastos, odontoblastos, fibroblastos y cementoblastos. Se observó los ameloblastos y odontoblastos columnares, cilíndricos (activos), con núcleos basales y su número se fue incrementando significativamente a través de los días (Figuras 1 y 2). Asimismo, predominó el tejido óseo maduro sobre el tejido osteoide y hubo una mayor presencia de bandas gruesas de fibras colágenas en el tejido periodontal, como se puede observar en las Figuras 3 y 4.
En el grupo B $\left(50 \%\right.$ de $\left.B_{2}\right)$ se observó una moderada población celular de ameloblastos, odontoblastos, fibroblastos y cementoblastos. Los ameloblastos y odontoblastos mostraban escasas microvacuolas. Presentaron un trabeculado óseo de grosor moderado, con predominio de tejido maduro sobre tejido osteoide, $y$ gruesas bandas de fibras colágenas en el tejido periodontal.

Con la dieta $25 \%$ de $\mathrm{B}_{2}$ (grupo C), se observó un trabeculado óseo delgado, con predominio de tejido osteoide sobre tejido óseo maduro; en el tejido dentoal- veolar hubo disminución de la población de ameloblastos, odontoblastos, fibroblastos, cementoblastos y escasa presencia de fibras colágenas.

El grupo $\mathrm{D}\left(0 \%\right.$ de $\left.\mathrm{B}_{2}\right)$ mostró una población celular disminuida de ameloblastos, odontoblastos, fibroblastos y cementoblastos, una cantidad muy escasa de fibras colágenas en el ligamento periodontal y el hueso alveolar muy adelgazado. Entre los cambios histomorfológicos observados en este grupo, se encontró pérdida de la polaridad celular, presencia de microvacuolas y áreas de pseudo-estra-

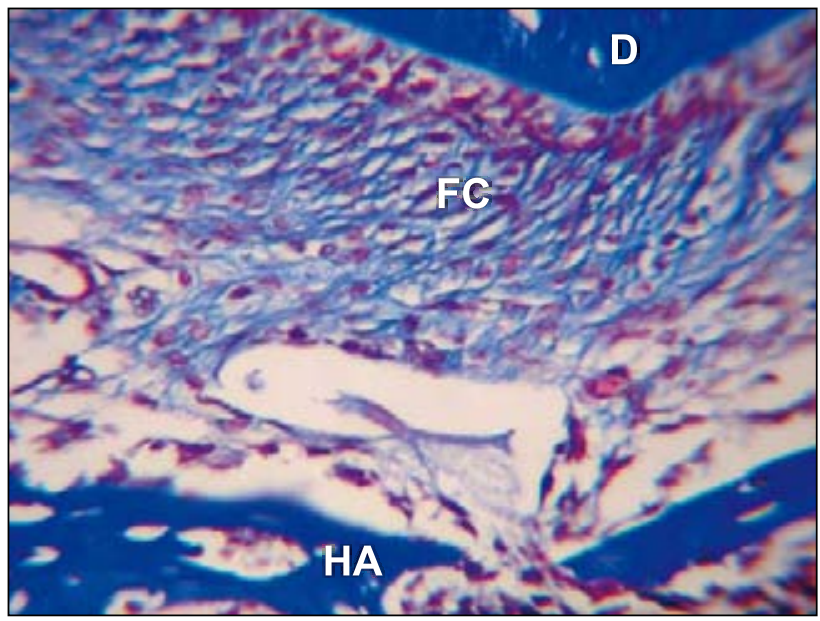

Figura 3. Fibras colágenas con $100 \%$ de riboflavina, a los 7 días. En corte coronal (40X) se observa en el tejido periodontal fibras de colágeno $(\mathrm{FC})$ de mayor espesor, situadas entre la dentina (D) radicular y el hueso alveolar (HA).

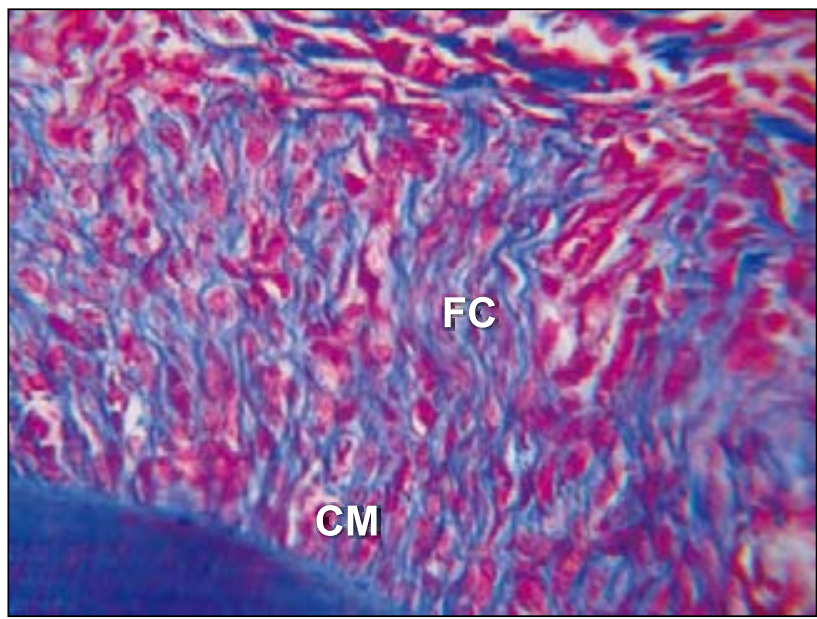

Figura 4. Fibras colágenas con $100 \%$ de riboflavina, a los 21 días. En corte coronal (40X) se observa en el tejido periodontal gruesas bandas de fibras colágenas $(\mathrm{FC})$, en mayor número, lineales y ondulantes, que se relacionan con el cemento $(\mathrm{CM})$ radicular. 


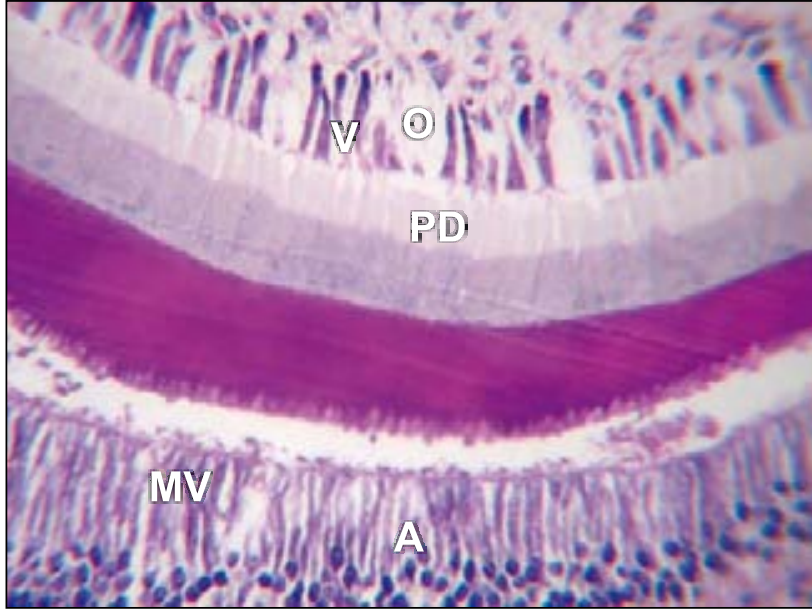

Figura 5. Tejido dentoalveolar con $0 \%$ de riboflavina, a los 7 días. En el corte coronal de diente (40X) se observa ameloblastos (A) en pseudo estratificación y con microvacuolas (MV). Los odontoblastos (O), con áreas vacuoladas (V). La predentina (PD) más clara, por el fenómeno de vacuolización.

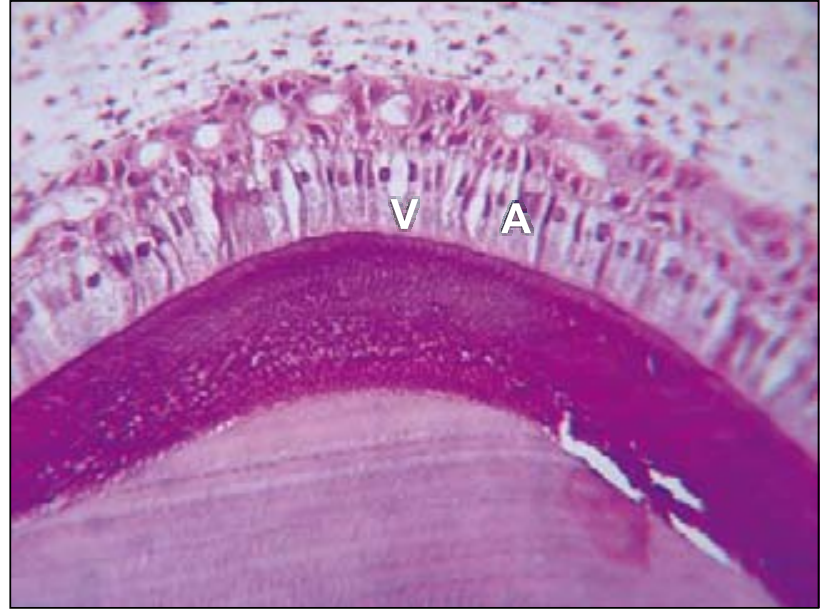

Figura 6. Tejido dentoalveolar con $0 \%$ de riboflavina, a los 21 días. Se observa (40X) en corte coronal a nivel de diente ameloblastos (A) afectados con muchas vacuolas $(V)$, en áreas en formas de grietas y en otras de microvacuolas. En los extremos, se ve inicio de pseudoestratificación. tificación en ameloblastos y odontoblastos (Figuras 5 y 6), aparición de edema en predentina, disminución de células activas de fibroblastos y cementoblastos. Además hubo notable disminución y adelgazamiento de las fibras colágenas, como se puede apreciar en las Figuras 7 y 8 . Asimismo, hubo reducción del grosor de las trabéculas óseas, con predominio de tejido osteoide sobre tejido óseo maduro y una médula ósea pobre en la serie mieloblástica y rica en tejido adiposo.

La diferencia que presentó el grupo A $\left(100 \%\right.$ de vitamina $\left.B_{2}\right)$ en cuanto al número de ameloblastos presentes fue significativa, con respecto a los grupos $\mathrm{B}$ ( $50 \%$ de $\left.\mathrm{B}_{2}\right), \mathrm{C}\left(25 \%\right.$ de $\left.\mathrm{B}_{2}\right)$ y $\mathrm{D}\left(0 \%\right.$ de $\left.\mathrm{B}_{2}\right)$, desde los 0 días hasta los 28 días $(p=0,000)$. Al observar los perfiles que describen el promedio de ameloblastos, se aprecia que los animales que consumieron $0 \%$ y $25 \%$ de $B_{2}$, sus crías tuvieron menor número de ameloblastos comparados a los grupos del $100 \%$ y $50 \%$ de vitamina $B_{2}$ (Figura 9 ).

En cuanto a la cantidad de odontoblastos presentes, la diferencia que presentó el grupo A ( $100 \%$ de vitamina $\left.B_{2}\right)$ fue significativa con respecto al grupo $\mathrm{D}$
( $0 \%$ de vitamina $\mathrm{B}_{2}$ ), desde los 0 días hasta los 28 días $(p=0,001)$. Al observar los perfiles que describen el promedio de odontoblastos, se aprecia que, a partir del día 14 en adelante, el grupo C disminuyó significativamente, acercándose al número de odontoblastos que presentó el grupo D; mientras que, en el grupo B se incrementó hasta los 28 días, con cantidades muy próximas a las del grupo control grupo A (Figura 10).

Respecto al número de fibroblastos presentes, la diferencia que presentó el grupo A $\left(100 \%\right.$ de vitamina $\left.B_{2}\right)$ fue sig-

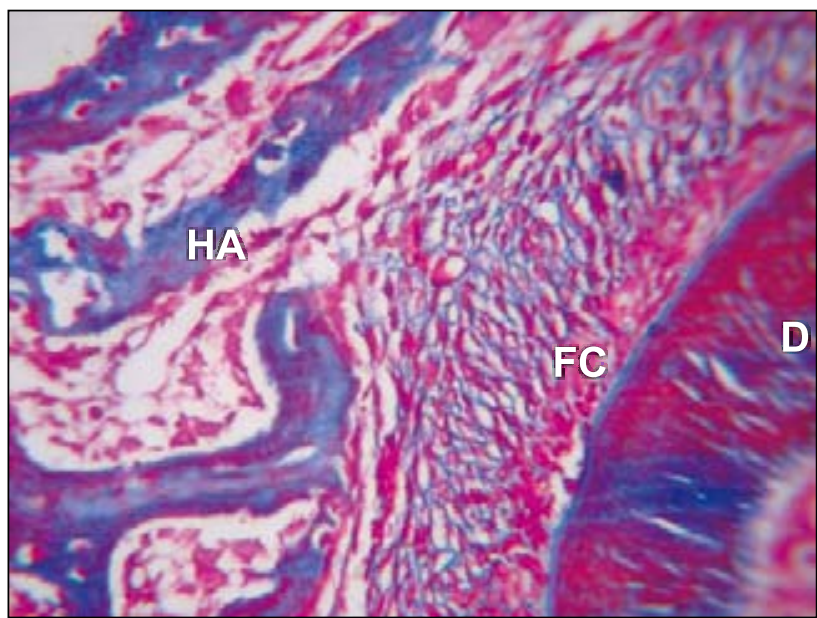

Figura 7. Fibras colágenas con $0 \%$ de riboflavina, a los 7 días. En corte coronal (40X), se observa en el tejido periodontal disminución de fibras colágenas (FC) delgadas entre la dentina (D) radicular y el hueso alveolar (HA).

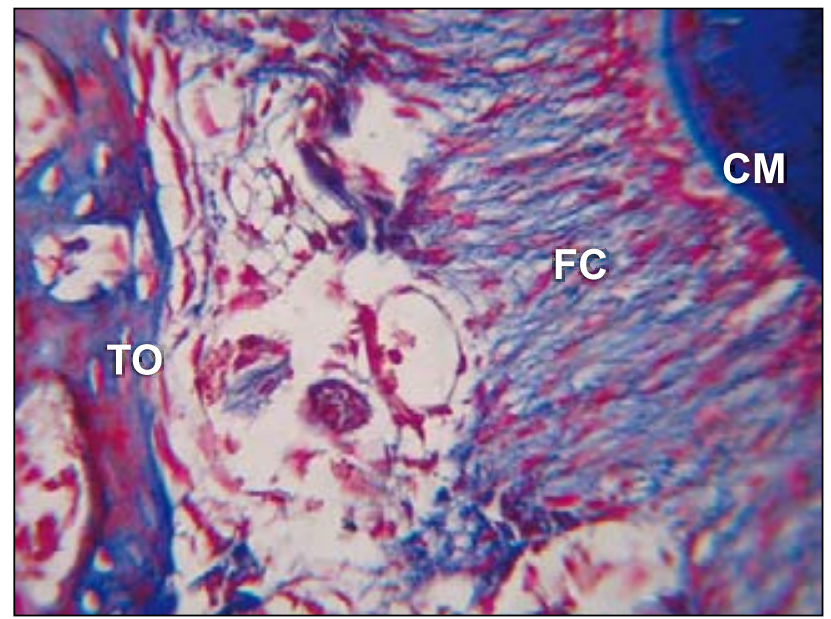

Figura 8. Fibras colágenas con $0 \%$ de riboflavina, a los 21 días. En corte coronal (40X), se observa en el tejido periodontal fibras colágenas $(\mathrm{FC})$ en menor cuantía y de menor grosor, que se ponen en contacto con tejido óseo (TO) delgado y cemento lineal (CM). 


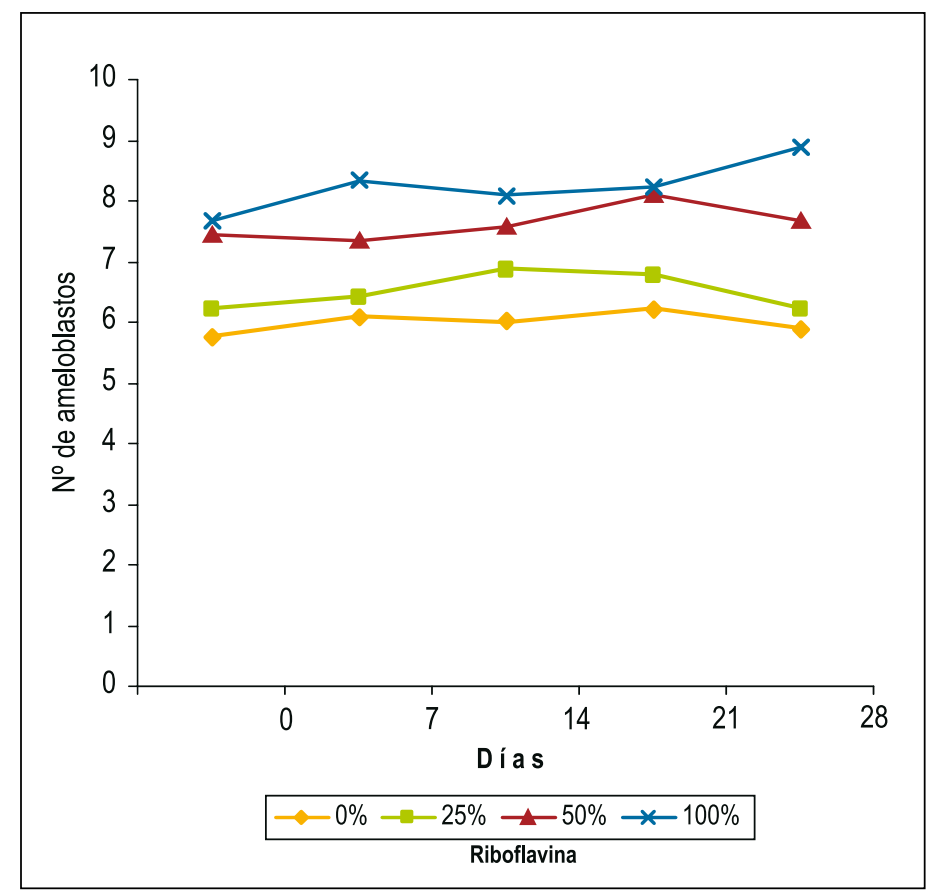

Figura 9. Promedio de ameloblastos en la progenie de ratas alimentadas de 0 a 28 días, en relación a dietas con diferentes proporciones de riboflavina.

nificativa respecto al grupo $B(50 \%$ de vitamina $\left.\mathrm{B}_{2}\right), \mathrm{C}\left(25 \%\right.$ de vitamina $\left.\mathrm{B}_{2}\right)$ y al grupo $\mathrm{D}\left(0 \%\right.$ de vitamina $\left.\mathrm{B}_{2}\right)$, desde los 0 días hasta los 28 días $(p=0,000)$, tal como se puede observar en la Figura 11; con lo que podemos afirmar que el consumo dietario de vitamina $\mathrm{B}_{2}$ está relacionado con el número de fibroblastos.

La diferencia que presentó el grupo A $\left(100 \%\right.$ de vitamina $\left.B_{2}\right)$ en cuanto a cantidad de cementoblastos presentes fue significativa respecto al grupo $\mathrm{B}(50 \%$ de vitamina $\left.B_{2}\right), C\left(25 \%\right.$ de vitamina $\left.B_{2}\right)$ y al grupo $\mathrm{D}\left(0 \%\right.$ de vitamina $\left.\mathrm{B}_{2}\right)$, desde los 0 días hasta los 28 días $(p=0,001)$, siendo más notoria la diferencia entre el grupo A y $B$ con relación al grupo $C$ y $D$ desde los 7 días (Figura 12).

La cantidad de fibras colágenas observadas en el tejido periodontal fueron significativas $(p=0,001)$, al analizarlas por medio de los porcentajes de vitamina $\mathrm{B}_{2}$ consumidas por los diferentes grupos a través de los días, y también por medio de la interacción de ambos factores. Al observar la Figura 13, apreciamos que el grupo $A\left(100 \%\right.$ de vitamina $\left.B_{2}\right)$ tuvo mayor cantidad promedio de fibras colágenas respecto a los grupos B, C y D, de acuerdo a la dieta consumida. los grupos $\mathrm{C}\left(25 \%\right.$ de vitamina $\left.\mathrm{B}_{2}\right)$ y $\mathrm{D}$ $\left(0 \%\right.$ de vitamina $\left.B_{2}\right)$, desde los 0 a 28 días $(p=0,000)$ (Figura 14).

\section{DISCUSIÓN}

El presente estudio tuvo como objetivo demostrar el efecto de la deficiencia y carencia de riboflavina sobre el crecimiento y desarrollo del tejido dentoalveolar de las crías de ratas, durante el periodo de la gestación y lactancia, comprobándose que existen cambios histomorfológicos en las células de este tejido, así como alteraciones del número de células presentes, la cantidad de fibras colágenas y el grosor del hueso alveolar en el tejido periodontal. A nivel celular, se observó perdida de polaridad nuclear, microvacuolas citoplasmáticas, grietas, áreas de pseudoestratificación.

Hubo un marcado retardo de crecimiento corporal, así como alopecia, alteraciones cutáneas y degeneración del tejido ocular, tal y como se comunica en algunos estudios ${ }^{(12-14)}$.

Las manifestaciones clínicas en seres humanos por deficiencia de vitamina B2 son debilidad, fatiga, dolor y sensibilidad
La diferencia que presentó los grupos A $\left(100 \%\right.$ de vitamina $\left.B_{2}\right)$ y B $(50 \%$ de vitamina $B_{2}$ ) en cuanto a nivel de grosor del hueso alveolar en el tejido dentoalveolar fue significativa respecto

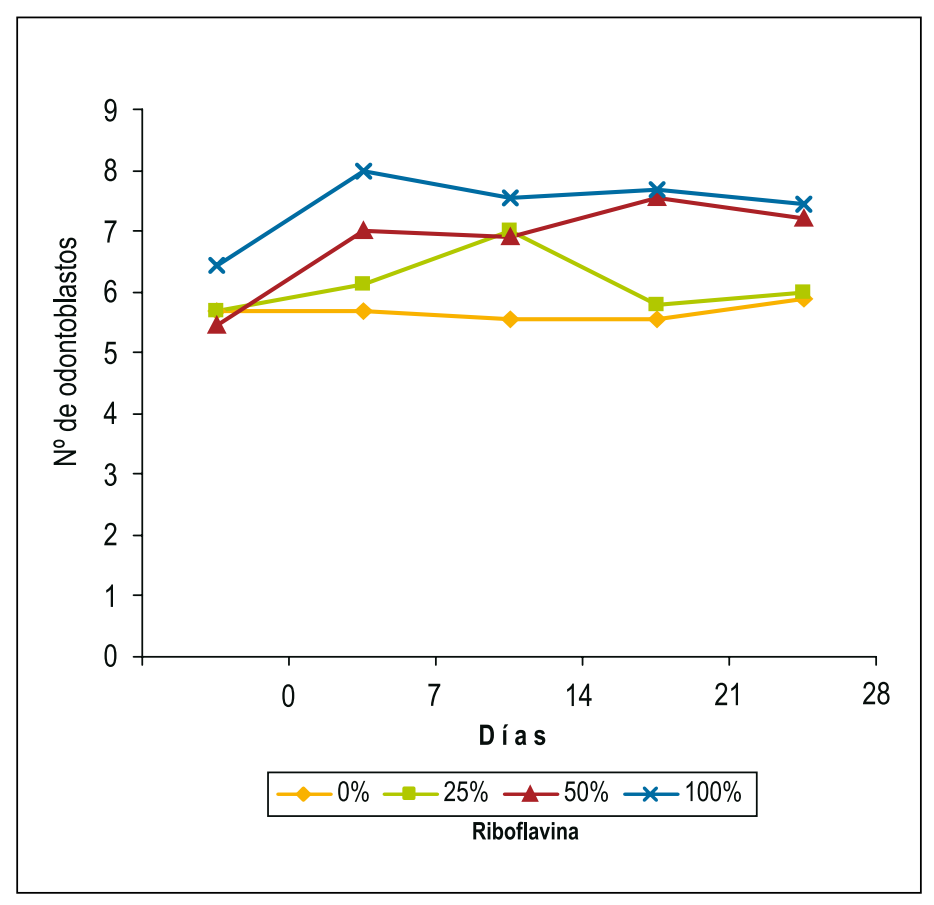

Figura 10. Promedio de odontoblastos en la progenie de ratas alimentadas de 0 a 28 días, en relación a dietas con diferentes proporciones de riboflavina. 


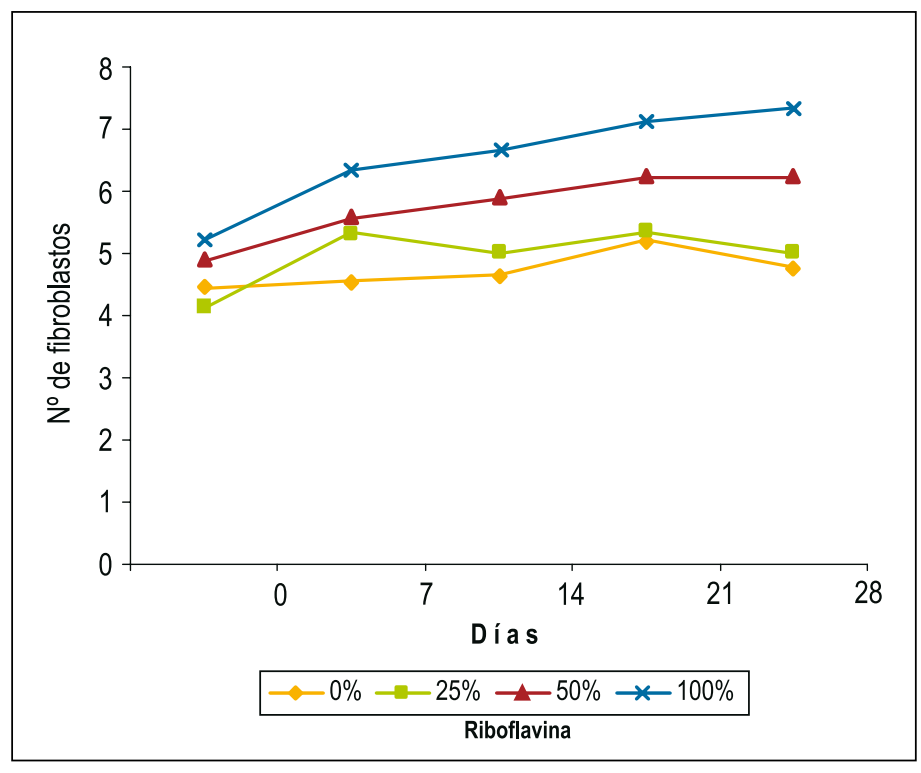

Figura 11. Promedio de fibroblastos en la progenie de ratas alimentadas de 0 a 28 días, en relación a dietas con diferentes proporciones de riboflavina.

en la boca, fotofobia, lagrimeo, sensación de quemazón y prurito en los ojos, y posibles cambios en la personalidad. La deficiencia extrema puede producir queilosis, estomatitis angular, dermatitis, erupción grasosa de la piel de los pliegues nasalabiales, escroto o vulva; una lengua púrpura e hinchada, desarrollo excesivo de capilares alrededor de la cornea ocular y neuropatía periférica ${ }^{(15)}$. Se sabe que la deficiencia de vitamina $B_{2}$ aparece en combinación con déficit de otras vitaminas hidrosolubles, como la tiamina y la niacina. De hecho, los síntomas clásicos, como la glositis y la dermatitis, pueden deberse a otras deficiencias asociadas.

En el presente estudio, se observó que las crías del grupo D (0\% de vitamina $B_{2}$ ) manifestaron un severo retardo en el crecimiento y una variedad de lesiones cutáneas con respecto al grupo control

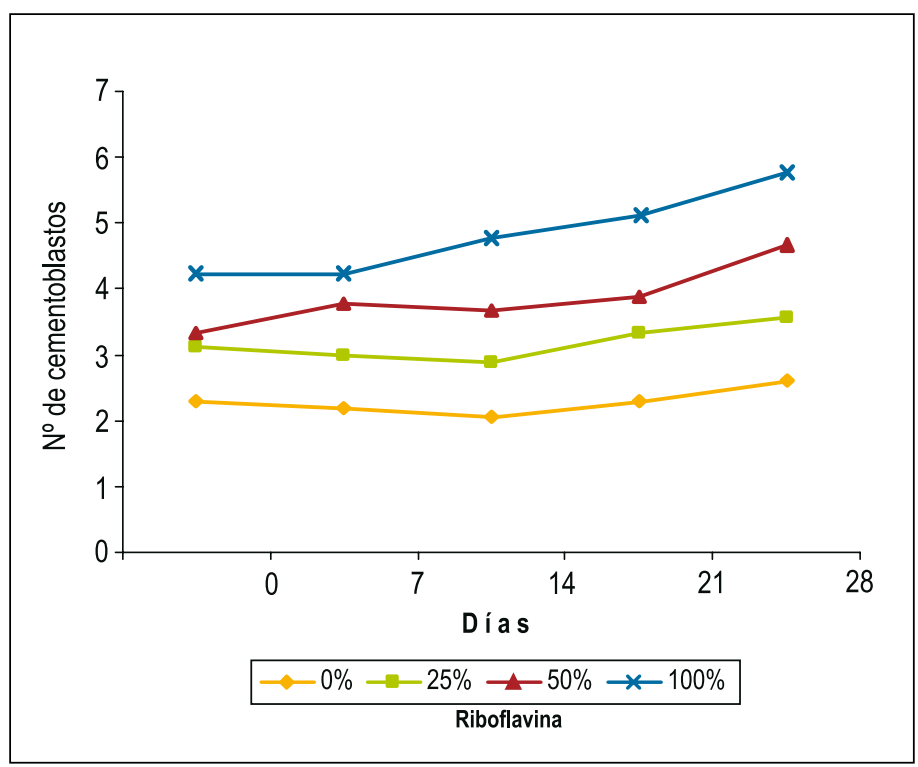

Figura 12. Promedio de cementoblastos en la progenie de ratas alimentadas de 0 a 28 días, en relación a dietas con diferentes proporciones de riboflavina.

$\left(100 \%\right.$ de vitamina $\left.B_{2}\right)$. Esto demuestra el efecto negativo que tiene la carencia total de vitamina $\mathrm{B}_{2}$ sobre el crecimiento de los animales. Las lesiones de la piel por deficiencia de vitamina $B_{2}$, según Prasad ${ }^{(16)}$, podrían deberse al déficit de la coenzima flavin mononucleótido (FMN), que interviene en el entrecruzamiento del colágeno.

Los resultados del promedio de peso corporal obtenido en las ratas del presente trabajo concuerda con los obtenidos por Patterson y Bates ${ }^{(17)}$, quienes encontraron que los pesos de las ratas con deficiencia de vitamina $\mathrm{B}_{2}$ mantienen diferencias significativas menores al compararse con el grupo control que contiene dieta de vitamina $B_{2}$ en proporciones adecuadas a sus requerimientos.

En la longitud corporal, se observó diferenciassignificativas en la talla promedio de los grupos de ratas A, B y C, respecto al grupo $\mathrm{D}$, donde el incremento de la talla en este último grupo fue lento y en menor proporción, indicando que las concentraciones de riboflavina interactúan significativamente en la longitud de las crías. Los trabajos de Roughead y Mc Cormick ${ }^{(18,19)}$ demuestran que la leche contiene niveles altos de flavina, con predominio de flavin adenin dinucleótido (FAD) y de riboflavina libre, que cubren los requerimientos de los lactantes y además pueden ser almacenados en los tejidos para posteriormente poder ser removidos en el caso de ser requeridos.

Respecto a la longitud de la mandíbula, hubo diferencias significativas entre los grupos A y B respecto a los grupos $\mathrm{C}$ y $\mathrm{D}$, siendo más notoria la diferencia entre el grupo control A y el grupo D. Una explicación de este efecto sería que las células mesenquimales no se histodiferencian en osteoblastos por la reducción de energía en forma de ATP; y dado que en la célula habría escasez de FM y FAD, el transporte de electrones para formar energía se encontraría limitado ${ }^{(20,21)}$. Para el mecanismo de histodiferenciación de las células mesenquimales, se necesita energía (ATP) y otros nutrientes, como calcio, ión fosfato y vitaminas. En cuanto al grosor de la mandíbula, hubo una diferencia significativa entre el grosor del grupo A y el grupo D. El periostio 


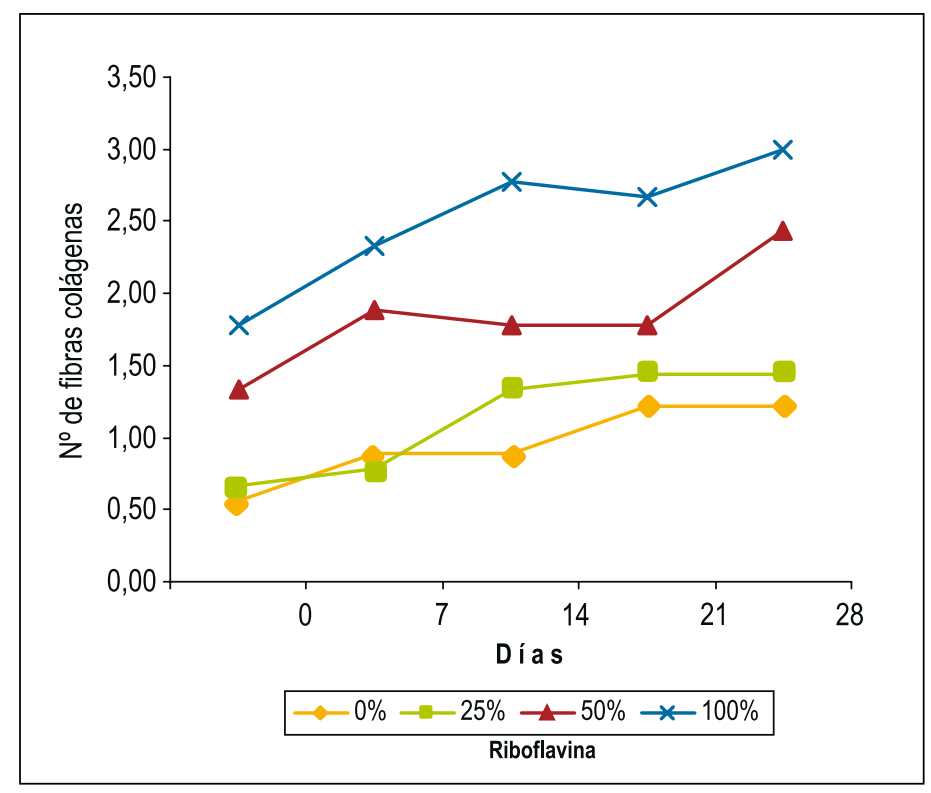

Figura 13. Promedio de fibras colágenas en la progenie de ratas alimentadas de 0 a 28 días, en relación a dietas con diferentes proporciones de riboflavina.

participa en el crecimiento periférico y aumenta el tamaño del arco mandibular, mediante aposición de laminillas en la superficie externa y resorción en la interna. Como este último mecanismo es más lento que el anterior, la mandíbula aumenta en espesor ${ }^{(22)}$.

Al observar los ameloblastos en el tejido dentoalveolar, se vio que las dos variables - los días y el porcentaje de riboflavina- interactúan significativamente en la formación de ameloblastos. La variabilidad entre los grupos experimentales (B-C-D) en relación al grupo A fue significativa. Esto nos permite deducir que la riboflavina tiene influencia sobre la cantidad de ameloblastos, relacionándose directamente con la capacidad que tienen las células del epitelio interno del esmalte (precursoras de los ameloblastos) en histodiferenciarse en ameloblastos. Al hacer el análisis histológico del efecto de la carencia de riboflavina (grupo D, $0 \%$ de vitamina $\mathrm{B}_{2}$ ), o deficiencia (grupo C, $25 \%$ de vitamina $B_{2}$ ) sobre los ameloblastos, se encontró que estas células están muy afectadas con la pérdida de su polaridad nuclear, la presencia de microvacuolas citoplasmáticas, grietas y áreas de pseudoestratificación, que indican una sustancial disminución de la actividad metabólica de estas células. Durante la etapa de mayor desarrollo dental es cuanlveolar, se vio el efecto significativo de la riboflavina. Esto nos permite deducir que do los ameloblastos se vuelven activos (cilíndricos), produciéndose el esmalte. Ten Cate ${ }^{(23)}$ refiere que el esmalte producido varía según la salud de la madre durante las etapas prenatales o de la salud en humanos después de nacer.

En los odontoblastos del tejido dentoa- la riboflavina está asociada a la cantidad y calidad de odontoblastos, relacionándose directamente con la capacidad que tienen las células ectomesenquimales en diferenciarse en odontoblastos. Durante el embarazo se induce la formación de proteínas transportadoras de riboflavina en varias especies, incluyendo la rata ${ }^{(24)}$. Al realizar el análisis histológico del efecto de la carencia ( $0 \%$ de vitamina $\mathrm{B}_{2}$ ), o deficiencia ( $25 \%$ de vitamina $B_{2}$ ) sobre el odontoblasto, se encontró que estas células se encuentran muy afectadas con evidentes áreas vacuoladas. Además, hay pérdida de su polaridad nuclear, edema de la predentina y áreas de pseudoestratificación, que indican una sustancial disminución de la actividad metabólica de estas células. Estos efectos podrían ser atribuidos a la falta de acción de la vitamina $\mathrm{B}_{2}$ en los odontoblastos ${ }^{(3)}$.

Al observar los fibroblastos en el tejido dentoalveolar, se vio que, durante el crecimiento y desarrollo, las dos variables -días de experimentación y concentraciones de vitamina $B_{2}$, están relacionadas con la formación de fibroblastos en el tejido dentoalveolar. Los grados de diferencia entre los grupos experimentales (B-C-D) respecto al grupo A (control) fueron significativos. Al hacer el análisis histológico del efecto de la carencia $(0 \%$

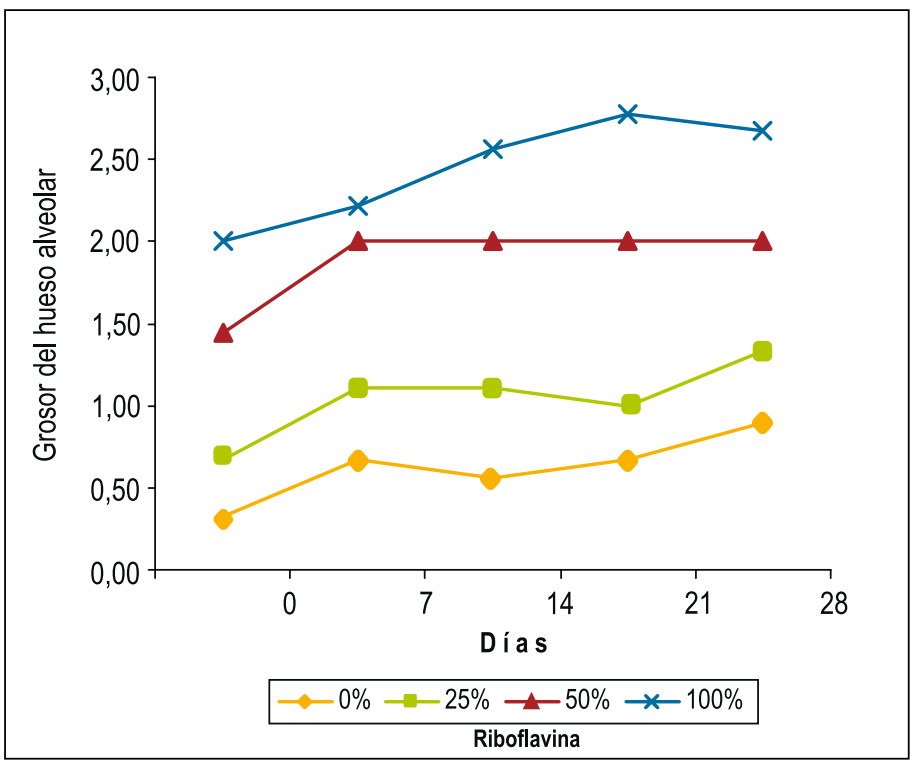

Figura 14. Promedio de hueso alveolar en la progenie de ratas alimentadas de 0 a 28 días, en relación a dietas con diferentes proporciones de riboflavina. 
de vitamina $B_{2}$ ), o deficiencia ( $25 \%$ de vitamina $B_{2}$ ) sobre los fibroblastos, se encontró escasos fibroblastos activos (fusiformes), con segregación de fibras colágenas, mayor presencia de fibroblastos jóvenes (no diferenciados) rodeados de histiocitos y de neocapilares. Hubo fibroblastos adultos anchos no formadores de fibras colágenas; esto nos indica una sustancial disminución de la actividad metabólica de estas células. Al respecto Gartner y Hiat ${ }^{(25)}$ mencionan que, en el ligamento periodontal, la remodelación del colágeno se logra por medio de una sola célula, el fibroblasto, que es capaz de sintetizar y degradar. Debido a la excepcional alta velocidad de recambio de colágeno en el ligamento periodontal, cualquier interferencia en la función de los fibroblastos por enfermedad o por desnutrición produce rápidamente una pérdida de los tejidos de sostén del diente.

En los cementoblastos del tejido dentoalveolar se vio que el efecto que tuvo la riboflavina sobre los cementoblastos en relación a las concentraciones de riboflavina fue significativo. Al hacer el análisis histológico del efecto de la carencia de vitamina $B_{2}(0 \%)$ o deficiencia de vitamina $B_{2}(25 \%)$ sobre el cementoblasto, se encontró formas aplanadas y atípicas y cementoblastos activos en menor cuantía. Al respecto, Ten Cate ${ }^{(23)}$ refiere que los cementoblastos, cuando están activos, son células cúbicas, formadoras de cemento radicular.

Se observó, en las fibras colágenas del tejido dentoalveolar, que una ingesta adecuada de vitamina $B_{2}(100 \%)$ en la rata madre conlleva a que las crías tengan una cantidad más elevada de fibras colágenas y de mayor grosor en el tejido periodontal, a diferencia de los otros grupos con deficiencia $(25 \%)$ o ausencia $(0 \%)$ de riboflavina. Esto condiciona a la capacidad que tienen las células productoras de colágena (fibroblastos) para sintetizar dichas fibras en la matriz periodontal. Se sabe que la colágena tipo I es la forma más abundante, representa alrededor de 90\% de colágena del cuerpo y que se encuentra en casi todos los tejidos conectivos, dermis, tendones, hueso, incluyendo el tejido dental ${ }^{(26)}$.
Respecto al hueso alveolar del tejido dentoalveolar, en el grupo A (100\% de vitamina $B_{2}$ ) hubo un mayor grosor, a diferencia de los otros grupos con una deficiencia o ausencia de ingesta de vitamina $\mathrm{B}_{2}$ en la dieta de las ratas.

En el grupo D, se pudo observar que a partir del día 21 existe una tendencia a aumentar de grosor en un porcentaje mucho mayor que en los otros días $(170 \%)$. Esto se explicaría porque las células ectomesenquimales ubicadas en el tejido periodontal, para diferenciarse a osteoblastos, en el grupo A lo harían más tempranamente que los del grupo $\mathrm{D}$ y C; por lo que, su ciclo de crecimiento lineal varía en ambos grupos. Otra posible explicación seria que en una síntesis limitada de ATP por carencia de vitamina $B_{2}$ para la formación de fibras colágenas, proteoglucanos y glucoproteínas, se produce una actividad osteoblástica retardada en los grupos $\mathrm{D}\left(0 \%\right.$ de vitamina $\left.\mathrm{B}_{2}\right)$, para la formación de hueso, lo que trae como consecuencias variaciones en el grosor del hueso alveolar.

Concluimos del presente trabajo que la carencia de riboflavina en la dieta durante la gestación y lactación produce cambios morfológicos en el tejido dentoalveolar de las crías de las ratas, provocando las siguientes características: pérdida de polaridad nuclear, presencia de microvacuolas citoplasmáticas y áreas de pseudoestratificación en ameloblastos y odontoblastos; disminución del número de células del tejido dentoalveolar; edema en predentina, por el fenómeno de vacuolización; células activas en menor cuantía en fibroblastos y cementoblastos; notable disminución de fibras colágenas y de menor grosor; trabéculas óseas disminuidas en su espesor, con mayor predominio de tejido osteoide; medula ósea pobre en la serie mieloblástica y con abundante tejido adiposo.

Así mismo, la carencia total de riboflavina en la dieta de las ratas durante la gestación y lactancia produjo en las crías una cantidad menor de ameloblastos, odontoblastos, fibroblastos, cementoblastos; escasa cantidad de fibras colágenas y un grosor de hueso alveolar muy delgado en el tejido dentoalveolar.

\section{REFERENCIAS BIBLIOGRÁFICAS}

1. McDonald L. Nutrición y sus trastornos [traducción Elena Cazenade Tapia]. 2a Edición. México DF, Santa Fé de Bogotá: Manual Moderno S.A. 1993.

2. Powers HJ, Bates CJ. A simple fluorimetric assay for pyridoxamine phosphate oxidase in erythrocyte haemolyscetes: effects of riboflavin supplementation and of glucose 6-phosphate dehydrogenase deficiency. Hum Nutr Clin Nutr. 1985;39:107-15.

3. Rivlin RS. Disorders of vitamin Metabolism: deficiencies, metabolic abnormalities and excesses. In: Wyngarden JB, Smith LH Jr, Bennett JC, Plum F, eds. Cecil's Textbook of Medicine. 19th ed. Philadelphia: WB Saunders; 1991. p. 1170-83.

4. Anderson LMV, Dibble M, Turkki P, Mitchell H. Nutrición y Dieta de Cooper [traducción José Pecina Hernández]. 17ma edición. México: Editorial Interamericana; 1987. p. 151-3.

5. Bates CJ. Human riboflavin requirements and metabolic consequences of deficiency in man and animals. World Rev Nutr Diet. 1987;50:215-65.

6. Shafer WG, Levy BM.Tratado de patología bucal [traducción María de Lourdes Hernández]. 4ta edición. Mexico: Editorial Interamericana; 1989. p. 676-7.

7. Wada Y, Kondo H, Itakura C. Peripheral neuropathy of dietary riboflavin deficiency in racing pigeons. J Vet Med Sci. 1996 Feb;58(2):161-3.

8. Ayala G, Vílchez W. Las Repercusiones del los Períodos Agrícolas y los Pisos agro ecológicos del ande Peruano, sobre el consumo de alimentos y estado nutricional de la población. 1ra edición. Lima: Empresa Científica Mundi; 1996.

9. Tomarelli M, Bernhart FW. A salt mixture supplying the national research council estimates of mineral requirements of the rat. J Nutr. 1966;89:495-6.

10. Méndez J. Effect of acute starvation and refeeding on body composition of rats fed previously at different levels of dietary protein. J Nutr. 1966;89:513-9.

11. Montgomery DC. Diseño y análisis de experimentos [traducido por Lic. Jaime Delgado Saldivar]. México D.F.: Grupo Editorial Iberoamericana S.A.; 1991. p. 45-52.

12. Campodonico C. La Riboflavina en el crecimiento óseo de ratas albinas. Tesis para optar el grado de Magíster. Facultad de Odontología, UNMS. 1997.

13. Delgadillo J, Ayala G. El Desarrollo Histológico del tejido óseo en Carencia de Riboflavina. Rev Odontología San Marquina. 2003;6(12):30-6.

14. Duerden JL. Bates of riboflavin deficiency on lipid metabolism of liver on brown adipose tissue of suckling rat pups. Br J Nutr. 1985;53:107-15.

15. Kathleen-Mahan L, Escott-Stump S. Nutrición y dietoterapia de Krause. 10ma edicion. México DF: Editorial Mc Graw- Hill Interamericana; 2003. p. 94-6. 
16. Prasad R, Lakshmi AV, Bamji MS. Impaired collagen maturity in Vitamina B2 y deficiency. Probale molecular bases of skin lesions. Biochem Med. 1983;30:333-41.

17. Patterson B, Bates C. Riboflavin deficiency,metabolic rat and brown adipose tissue function in suckling and nearnling rats. Br J Nutr. 1989;61:47583.

18. Roughead ZK, McCormick DB. Flavin composition of human milk. Am J Clin Nutr. 1990;52(5):85457.

19. Roughead ZK, McCormick DB. Qualitative and quantitative aseessment of flavins in cows milk. J Nutr. 1990;120(4):382-8.

20. Leninger AL, Nelson DI, Cox MM. Principios de bioquímica [traducido por Fernando Calvet Prats]. 2da Edición. Barcelona, España: Editora Omega S.A.; 1995. p. 352-6.
21. Murray R, Mayes A, Peter A. Harper Bioquímica llustrada. 16va edición. México DF: Editorial El manual Moderno; 2004. p. 545-7.

22. Abramovich A. Embriología de la región máxilo facial. 3ra edición. Madrid, España: Editorial Médica Panamericana; 1997. p.192-207.

23. Ten Cate AR. Histología oral: desarrollo, estructura y función. 2da Edición Buenos Aires: Editorial Medica Panamericana; 1994. p. 146-70,191232.

24. Muniyappa K, Adiga PR. Isolation and characterization of Riboflavin- binding Protein from pregnant-rat. Serum Biochem J. 1980;186:53740.

25. Gartner LP, Hiatt LJ. Histología, texto y atlas [traducción Dr. Santiago Sapiña]. Renard. 1ra edición en español. México DF: MC Graw- Hill Interamericana; 1997 p. 119-35,321-7
26. Leeson TS, Paparo A. Texto, Atlas de histología. México DF: MC Graw Hill Interamericana; 1990. p. 130-4,159-88.

Manuscrito recibido el 18 de febrero de 2009 y aceptado para publicación el 30 de marzo de 2009.

\section{Correspondencia:}

Dra. Juana Delgadillo Avila

Calle Ramón Cerdeira 175, San Borja

Lima 41, Perú

Correo-e: juanidelgadillo@hotmail.com 